\title{
Medicina Basada en Evidencia
}

\section{Reflexiones sobre la evidencia en medicina}

\author{
Félix Muñoz $C^{1}$, Fernando Cabrera $\mathbf{R}^{2}$. \\ Reflections on evidence-based \\ medicine
}

This is a review of the basic concepts of evidence, medicine, evidence-based medicine and its effects. Evidence-based medicine is a contribution for statistical design and management, that allows the gathering of scientific information, but does not completely exclude bias. Its methodology proceeds with order and caution, generating a highly probable mass of knowledge. The quality of the information obtained from diverse sources such as meta-analysis to expert opinions is classified in different levels according to the «strength» of the evidence in which it is based. The best contribution of evidence-based medicine is probably for diseases of incidence and does not replace clinical experience and patientsphysician relationship. Basically we treat ill people and not illnesses. Without clinical experience, medical practice can be tyrannized by evidence-based medicine that can become inapplicable or inappropriate. Without evidence-based medicine, medical practice will not be updated, limiting our professional skills (Rev Méd Chile 2005; 133: 1252-8).

(Key Words: Bias (epidemiology; Evidence-based medicine; Meta-analysis)

Recibido el 10 de junio, 2005. Aceptado el 22 de junio, 2005.

${ }^{1}$ Servicio de Medicina Interna, Hospital Clínico San Francisco de Borja-Arriarán, Facultad de Medicina Universidad de Chile. ${ }^{2}$ Departamento de Pediatría, Facultad de Medicina, Universidad de Chile.

S endo la medicina una ciencia social, no debiera sorprender que se hable de Medicina Basada en la Evidencia (MBE). Esta expresión fue utilizada anteriormente cuando médicos como Pierre Louis, a fines del siglo XVIII, buscaron la verdad en la observación sistemática de los pacientes para rechazar la declaración de la autoridad que aconsejaba la venosección como tratamiento contra el cólera ${ }^{1}$. Esta expresión se ha consolidado recientemente, en 1992, cuando un grupo canadiense dirigido por Gordon Guyat la empleó para acoger y promover artículos de

Correspondencia a: Dr. Félix Muñoz C. Costa de Marfil 296. Santiago/Chile. Fono: 5446600. Fax 5446614.

E-mail: fmunoz@hsba.cl fmunoz@ctcinternet.cl investigación clínica y epidemiológica que cumplen con una base matemático-estadística que les satisface como «evidencia». Desde ese momento el número de comunicaciones sobre su práctica ha crecido exponencialmente, incluyendo revistas y textos ${ }^{2}$. La rápida expansión de la MBE no sólo es explicable por una necesidad de información sobre diagnóstico, pronóstico, terapia y prevención, carencias de las fuentes tradicionales (textos), limitaciones de tiempo al lado del paciente 0 de lectura, sino también como apoyo a las políticas de salud pública que creen poder contener costos, sustituyendo de paso al empirismo científico ${ }^{3}$.

Sin duda el desarrollo de la MBE ha tenido y tiene un gran impacto actual en su aporte sistemá- 
tico a la investigación y manejo clínico, llegando a hablarse de el nuevo paradigma médico $\Downarrow^{4}, 5$. Nuestro interés es analizar si estamos frente a la evidencia o certeza cartesiana o si se trata solamente de una herramienta útil para el conocimiento y el arte médico, contextualizando someramente los conceptos de evidencia, ciencia y medicina, la MBE y sus efectos.

\section{Sobre la EVIDENCIA}

Aspectos previos. El conocimiento, según Cassirer, es un proceso por el que elevamos a la conciencia una realidad ya de por sí existente, ordenada y estructurada 6 . Toda época posee además un sistema fundamental de conceptos y premisas generales y últimos, por medio de los cuales domina y ordena en unidad la variedad de la materia que la experiencia y la observación le suministran. Es necesario, en consecuencia, iluminar las formas de conocer, con sus ventajas y limitaciones, ante el peligro de la rigidez y la dependencia de la razón.

La palabra con que los griegos nombraban la verdad es alétheia, que quiere decir des-cubrir, quitar el velo. Para descubrir la realidad será necesario fabricarnos una realidad imaginaria y confrontarla con los hechos efectivos. Si calzan unos con otros es que hemos des-cubierto la realidad que los hechos cubrían. La ciencia cumple llevando a cabo esta doble tarea, una puramente imaginativa, creadora y otra confrontadora. Para Ortega, la ciencia es, en efecto, interpretación de los hechos ${ }^{7}$. Heidegger dirá que la ciencia es la teoría de lo real; entendiendo teoría como reelaboración y lo real lo actuante y lo actuado ${ }^{8}$.

El ejercicio profesional de la medicina requiere de las ciencias naturales y humanas para que el médico comprenda la enfermedad, al enfermo y su entorno, pero el dominio de ellas no capacita para el ejercicio de la profesión ${ }^{9}$. Como práctica, la medicina se estructura entre los saberes puros y el puro empirismo ${ }^{10}$. Laín Entralgo apunta a un saber-hacer $^{11}$. Un hacer impregnado de teoría. Un saber que se justifica en la acción. Un arte, donde además se conjuga un encuentro, una comunicación de mirada, rostro, lenguaje. El enfermo habla al médico como testigo e intérprete de su mundo, de su cuerpo, de su intimidad ${ }^{12}$.
En su ejercicio profesional el médico se enfrenta a múltiples interrogantes. Lo habitual es consultar a colegas expertos, libros, revisiones en revistas o en internet, que pueden resultar ineficaces o inducir a errores. Esto motivó al grupo de la Universidad de McMaster a iniciar un nuevo movimiento dentro de la enseñanza y práctica de la medicina, que no por azar denominaron «Evidence Based Medicine». El mejor significado de «evidence» no corresponde a «evidencia» castellana, sino más bien a prueba, de modo que una mejor traducción del título debiera ser «Medicina Basada en Pruebas $\rangle^{13}$. Los propios autores se refieren a ella como «a utilización consciente, juiciosa y explícita de las mejores pruebas disponibles, en la toma de decisiones sobre el cuidado de los pacientes».

Evidencia cartesiana. René Descartes (1596-1650), matemático, físico, abogado, médico y filósofo, la figura más destacada sin lugar a dudas de una generación decisiva (que incluye a Galileo y William Harvey entre otros), en el advenimiento de la filosofía y la ciencia moderna, es quien en dos de sus obras, «eglas para la dirección del espíritu» y el Discurso del Método $\$ 4,15$, se preocupa de encontrar un modo de conocer que impida el error y la subjetividad. Entiende por método, reglas ciertas y fáciles, mediante las cuales el que las observe exactamente no tomará nada falso por verdadero, y no empleando inútilmente ningún esfuerzo de la mente, sino aumentando siempre gradualmente su ciencia, llegará al conocimiento verdadero de todo aquello de que es capaz»(Regla IV) ${ }^{14}$. Su aplicación sería extensible a cualquier nuevo campo del saber, permitiendo aumentar los conocimientos y descubrir verdades nuevas. El método cartesiano no expresa el lugar común de que una ciencia debe tener su método, sino el procedimiento o modo como estamos tras la cosa.

Descartes no acepta como verdadero sino lo que es «evidente», expresado en la I Regla del Discurso del Método: «No reconocer como verdadera cosa alguna que antes como tal no la hubiera evidentemente reconocido; es decir, evitar escrupulosamente la precipitación y la prevención, y no comprender en mis juicios nada más que lo que clara y distintamente se ofreciera a mi espíritu que nunca hallara ocasión de ponerlo en duda $»^{15}$. 
Aquí, lo evidente se produce sólo en la intuición, es decir, en un acto puramente racional por el que nuestro intelecto capta de modo inmediato una idea. Implica que no hay de por medio cadena deductiva y nada sensible. Descartes excluye los conocimientos que son únicamente probables. La certeza exige la desestimación absoluta de lo probable. Lo que no es claro y distinto (evidente) es confuso y oscuro, debiendo ser rechazado. La evidencia, como criterio de verdad, exige que el conocimiento se someta a sus propias leyes, independiente de lo que exista externamente a nuestra propia mente y su proceder. En otras palabras, se adecua la cosa al intelecto, ocupándonos de aquellos aspectos u objetos de los que nuestra razón pueda obtener un conocimiento indudable (Regla II) ${ }^{14}$, lo que podamos ver con claridad y evidencia (Regla III) ${ }^{14}$. La verdad de nuestras representaciones no es ya la cosa concreta, sino el postulado de la cohesión interior y de la ausencia de contradicciones ${ }^{6}$. Descartes dejaba así abierto el camino para el desarrollo de la ciencia moderna, resaltando su carácter de ciencia de hechos, ciencia experimental y de medición, agregándose una actitud intelectual fundamental de saber matemático ${ }^{16}$.

Medicina basada en la evidencia. La metodología de la MBE radica en un cuidadoso proceso que debe seguirse para llegar a la pretendida verdad de las pruebas o evidencias. Sus principales pasos son los siguientes:

Paso $1^{\circ}$ : El problema de la pregunta. Ha sido enunciada indistintamente, sea como sormular de manera precisa una pregunta a partir del problema clínico del paciente», formular preguntas clínicas pertinentes y contestables», o «convertir la duda en una pregunta clínica estructurada». Estas preguntas siempre se traducirán en una investigación de la información médica disponible en un área específica de la medicina. En el caso del paciente individual se trata de averiguar cuál es su problema principal, la mejor forma de estudiarlo y los efectos de los nuevos fármacos; ya sea frente al enfermo en sala de hospital, sala del consultorio de Atención Primaria o consulta privada. Lo mismo se lleva a cabo en actividades de investigación epidemiológica, terapéutica, etc. Siempre interesa buscar la bibliografía pertinente, hecho que se cumplirá en el segundo paso. El problema de la pregunta no es otra cosa que la conversión de la duda en una pregunta clara y distinta. Luego, dirigir la mente en dirección al problema más importante de la cosa investigada, el posible diagnóstico o el tratamiento más indicado.

En el problema de la pregunta podemos distinguir: primero, el origen de la pregunta, que puede ser el problema clínico, los ensayos de un fármaco nuevo, una investigación terapéutica, etc. Segundo, los objetivos de la pregunta, es decir, focalizar la búsqueda en aquellos artículos que puedan contestarla. Tercero, los componentes de la pregunta: a) tipo de paciente o población; b) el tipo de la intervención (un procedimiento diagnóstico, un tratamiento o uso de una droga, un método de investigación) c) el grupo de comparación (sujetos no intervenidos, grupo control) y, finalmente, d) el resultado.

Paso $2^{\circ}$ : El problema de la búsqueda de las pruebas o «evidences». Se ha enunciado de diferentes maneras, de modo que podemos decir que es docalizar las pruebas disponibles en la literatura», sastrear de manera sistemática los artículos relevantes», o «ealización de una búsqueda bibliográfica para encontrar artículos que puedan responderlas». Al igual que en el problema de la pregunta tenemos aquí diferentes aspectos que considerar en la búsqueda de la verdad: primero, análisis de los artículos, revisiones, puestas al día en la literatura disponible; en segundo lugar, recurrir a herramientas y estrategias que se han ido perfeccionando en el último tiempo.

Sin embargo, todavía la mayor parte de los resultados se obtienen por métodos tradicionales de revisiones con estadísticas poco rigurosas, cualitativas, incapaces de dar resultados fiables, cuando se trata de muchas variables o son numerosos los estudios publicados. El resultado se traduce en datos a veces contradictorios, negando o confirmando el mismo estudio.

La solución ha venido desde la estadística. En 1976 Gene Glass propuso un método que llamó meta-análisis (del griego meta= después de y analisis $=$ descripción, interpretación). Puede definirse como análisis estadístico integrador de una gran colección de resultados extraídos de trabajos individuales similares. Finalmente puede apelarse a comités de expertos que han sido creados para este objeto en universidades 0 instituciones dedicadas a la investigación. 
Paso $3^{\circ}$ : Evaluación crítica de las pruebas y resultados. Es la revisión detallada de los artículos seleccionados, de cada uno de los hallazgos, documentos recuperados o pruebas encontradas en la literatura disponible, incluyendo meta-análisis si hay disponibles. No es infrecuente encontrar errores en los trabajos de investigación publicados, sea por fallas en el diseño, tamaño de la muestra, uso de las pruebas de significación estadística, falta de uniformidad en la presentación de los resultados y alusión indebida en las conclusiones a los resultados de las pruebas de hipótesis ${ }^{17,18}$.

En el análisis de los resultados se debe considerar: a) Evaluar la validez de los resultados encontrados, esto es, su validez interna; b) Averiguar sus resultados y magnitud del efecto; c) Confrontar si los resultados ayudarán en la atención del paciente 0 en el problema que se enfrenta (validez externa).

Paso $4^{\circ}$ : El último paso de la MBE es llevar a la práctica las conclusiones de la evaluación realizada. Corresponde a su aplicación metódica y razonada, basada en pruebas, además de precisar diagnósticos, recurrir a procedimientos, o hacer uso de terapias en el paciente individual o en todos aquellos con iguales características.

Necesidad de graduar el nivel Evidencia según las pruebas disponibles. La exigencia en el diseño de la investigación, incorporando sistemáticamente modelos controlados, aleatorios, e incluso doble ciego han aportado junto con el meta-análisis un mayor grado de seguridad en la información obtenida, o evidencia de nivel I. Es una serdad» que se funda en la posibilidad de que aquello muy poco probable se considere como imposible, pasando por consiguiente desde una confianza objetiva a una confianza meramente subjetiva ${ }^{19}$. Limitaciones en efectuar investigaciones con grandes números, de diseños u otras, ha conducido a graduar el nivel de evidencia»según la calidad de las pruebas disponibles en grados I, II y III, o I, II, III, y IV según diversos grupos de trabajo 20,21 . Esta gradación o categorización de las pruebas (evidencias) dan paso a la denominada fuerza de las recomendaciones». Estas recomendaciones se estratifican como A, B, C, y D, correspondiendo el nivel A a un grado de evidencia I. De esta manera se ha llegado a disponer de un cuerpo de información cada vez mayor, en diversas ramas de la medicina, accesible a través de llaves, que permite obtener las mejores pruebas respecto a tópicos epidemiológicos y terapéuticos.

Ventajas y desventajas de la MBE. En términos epistemológicos, la MBE es un intento loable que busca aplicar en el área de la medicina la primera regla del Discurso del Método. No cabe duda de que su aporte nos entrega una información más confiable del «estado del arte» del conocimiento médico actualizado y su aplicabilidad, particularmente en entidades morbosas que presentan un mayor impacto dadas sus altas tasas de incidencia. Por otra parte, puede ser ejercida por médicos de variado nivel formativo con tal que dispongan de acceso a la información basada en pruebas. Se dice además que es democrática, abierta al debate, en contraposición a las que a veces tratan de imponer las figuras doctas de la medicina ${ }^{20}$.

Frente a lo anterior se levantan algunas críticas:

- La evidencia debe ser entendida como información basada en pruebas, ya que no corresponde a la evidencia cartesiana, aunque su metodología nos recuerde el Discurso del Método o las Regulae.

- Siempre existirá un sesgo y la posibilidad de superarla.

- Debe recordarse que el «médico trata enfermos y no enfermedades»y que la esencia del acto médico es la relación hombre enfermo/hombre médico, aspecto que sólo madura con la experiencia, no reemplazable por una mejor documentación.

- No siempre, especialmente en Atención Primaria, la literatura de la MBE tiene respuestas para su enfrentamiento. Su ámbito, que es ideal para abarcar patologías de gran incidencia, deja otras sin la posibilidad de estudios estadísticos refinados, en las que las ideas claras y distintas y la experiencia razonada puedan avanzar en su manejo.

- Finalmente se señala que su implantación arbitraria con fines economicistas sólo sería conducente a rebajar el poder de la autoridad médica basada en la experiencia y a coartar la libertad de las decisiones.

- La propuesta lógica actual consistirá en usar la mejor evidencia disponible para la toma de 
decisiones clínicas, sin desconocer la importancia de la experiencia ${ }^{22}$.

\section{CONCLUSIONES}

La MBE o mejor expresado en castellano Medicina basada en pruebas, es un aporte en el diseño y manejo estadístico que permite obtener información científica variada. Su metodología, que mira de cerca a Descartes, figura precursora de la ciencia moderna, procede con orden y medida, encontrándonos en distintos momentos de sus proposiciones con referencias explícitas e implícitas a la primera regla del Discurso del Método o a Las Reglas para la Dirección del Espíritu, generando en su accionar una masa de conocimientos actualizados altamente probables. Ellos pueden ser consultados mediante llaves, útiles para la aplicación en el caso individual de un paciente 0 colectivos, en la investigación epidemiológica,

\section{REFERENCIAS}

1. Wulf H, Pedersen SR, Rosemberg R. Introducción a la filosofía médica Ed. Triacastela. Madrid, 2002.

2. Sackett D, Straus S, Richardson WS, Rosemberg W, HAYNeS RB. Medicina basada en la evidencia. Cómo practicar y enseñar la MBE. Ed Harcourt, S.A. Versión en español de $2^{\mathrm{a}} \mathrm{Ed}$. de EvidenceBased-Medicine, 2000.

3. Cervera S, Viñes J. El ejercicio de la medicina en el contexto médico-social del año 2000. Rev Esp Salud Pública v.73 n1 Madrid Jan/Fev. 1999. http://www.scielosp.org/scielo.php?script=sci.

4. Evidence-Based Medicine Working Group: Evidence Based Medicine. A new approach to teaching the practice of medicine. JAMA 1992; 268: 2420-5.

5. Marecos E. Retroceder para avanzar. Revista de Posgrado de la VI Cátedra de Medicina № 114Abril 2002. http://www.google.cl/search?q=cache: N58NMrmBmV4J:med.ar/revista/ (Consultado el 20 de agosto de 2004)

6. CASSIRER E. El problema del conocimiento I, Ed. Fondo de Cultura Económica, S.A. México, DF, Quinta reimpresión, 1993.

7. Ortega y Gasset J. En torno a Galileo. Revista de Occidente, S.A. Madrid, 1959. comparación de terapias, etc. La calidad de la información obtenida hasta el presente, en razón de la perfección de sus diseños, desde el metaanálisis hasta las opiniones de expertos, ha sido llevada a grados de sevidence», lo que de paso ponen a la vista el estado del conocimiento científico médico en los diversos campos de su accionar.

Sin lugar a dudas la MBE es un gran aporte y permanecerá hasta ser superada en el futuro. Sin embargo, su aporte será menor en patologías de baja incidencia y de ningún modo podrá reemplazar la experiencia y la relación médico-paciente. Los «buenos médicos» deberán hacer uso de su experiencia clínica y la mejor «evidence» externa disponible. Sin la experiencia clínica la práctica profesional puede correr el riesgo de ser tiranizada por la MBE, ser inaplicable o inapropiada para un paciente individual. Sin la MBE la práctica profesional se encontrará atrasada, en detrimento de sus enfermos.

8. Heidegger M. Ciencia y Meditación. En Filosofía, ciencia y técnica. $4^{a}$ edición. Editorial Universitaria, Santiago de Chile, 1997.

9. Goic A. El fin dela medicina. Publicaciones Técnicas Mediteráneo Ltda. Santiago de Chile, 2000.

10. Lolas F. Hacia una teoría de la medicina como disciplina y como profesión. En Lolas F., Proposiciones para una teoría de la medicina. Editorial Universitaria S.A. Santiago de Chile. 1992.

11. Laín Entralgo P. Historia Universal de la Medicina. Barcelona Salvat, 1972.

12. Laín Entralgo P. Estructura de la relación entre el médico y el enfermo. En Laín Entralgo, El médico y el enfermo. Ediciones Guadarrama S.A. Madrid, 1969.

13. Bravo R, Campos C. Medicina basada en pruebas (Evidence-based Medicine) JANO (EMC) 1997; 53: 71-2.

14. Descartes R. Reglas para la dirección del espíritu. Traducción de Navarro Cordón J.M. Alianza Editorial, S. A. Madrid, 1984.

15. Descartes R. El discurso del método. Mestas ediciones, Madrid, 2000.

16. HeIDEgger M. La moderna ciencia natural matemática y la génesis de una crítica de la razón pura. En: La pregunta por la cosa. Editorial Memphis, Buenos Aires, 1992. 
17. SaRRIA M, Silva LC. Las pruebas de significación estadística en tres revistas biomédicas: una revisión crítica. Rev Panam Salud Pública/Pan Am J Public Health 2004; 15: 300-6.

18. Clark ML. Los valores $P$ y los intervalos de confianza: ¿en quien confiar? Rev Panam Salud Pública/Pan Am J Public Health 2004; 15: 293-6.

19. PÉREZ C. Sobre un concepto histórico de la ciencia. De la epistemología actual a la dialéctica. Universidad ARCIS. LOM Ediciones, 1998.
20. Guerra JA, Muñoz PM, Santos JM. Las revisiones sistemáticas, niveles de evidencia y grados de recomendación. Actualización 24/10/2003, www. fisterra.com (Consultado 19 de septiembre de 2004)

21. The National Collaborating Centre for Chronic Conditions. NICE guideline $\mathrm{N}^{\circ} 12$. Chronic obstructive pulmonary disease. Thorax 2004; 59 (Suppl I): 1-232.

22. LETELER LM, Moore P. La medicina basada en evidencia. Visión después de una década. Rev Méd Chile 2003; 131: 939-46. 